\title{
How we predicted the memristor
}

\author{
The memristor - a resistor with memory - was first postulated back in 1971, but it took nearly four decades \\ before it was experimentally confirmed. Leon O. Chua explains how he developed a nonlinear circuit theory in \\ which the memristor emerges naturally as the fourth basic circuit element.
}

\author{
Leon O. Chua
}

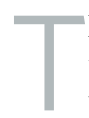

he dawn of nonlinear electronics was ushered in by newly minted twoterminal electronic devices bearing such intimidating monikers as the Esaki diode, Josephson junction, varactor, thyristor, the IMPATT (impact ionization avalanche transit-time) diode and the Ovonic threshold switch. The proliferation of these exotic devices in the 1960s was an exciting period for electronic engineers. At this time, I was working on my PhD, trying to make sense of these strongly nonlinear and dynamical electronic devices. Rather than charting a taxonomy to pigeonhole them, I opted for an axiomatic definition of a few basic nonlinear circuit elements that could be used to model a broad variety of nonlinear devices. I joined Purdue University upon graduation in 1964 and was asked to revamp its outdated circuit analysis curriculum, thereby providing me with an ideal launch pad for teaching nonlinear circuit theory through my deviceindependent 'black box approach'.

To analyse circuits made of strongly nonlinear and dynamical electronic devices, it is necessary to have realistic device models made of well-defined nonlinear circuit elements as building blocks. Such models did not exist back then because engineers were taught to overcome nonlinearities by expanding them in a Taylor series and then retaining only the linear term, which can neatly map into a linear circuit model. These models invariably gave rise to grossly inaccurate and misleading results. Unfortunately, the 'linearize then analyse' culture endowed upon electronic engineers of the day had made it impossible to devise any generalizations, owing in part to the lack of a circuit-theoretic foundation for basic nonlinear circuit elements. (Even Richard Feynman would sloppily use the old-fashioned name condenser, instead of capacitor, in his classic 1964 textbook, The Feynman Lectures on Physics.) Electronic circuit theory is concerned only with the prediction of the voltage $v(t)$ and current $i(t)$ associated with the external terminals of the linear and nonlinear electronic devices interconnected in a circuit, and not with the 'internal' physical variables associated

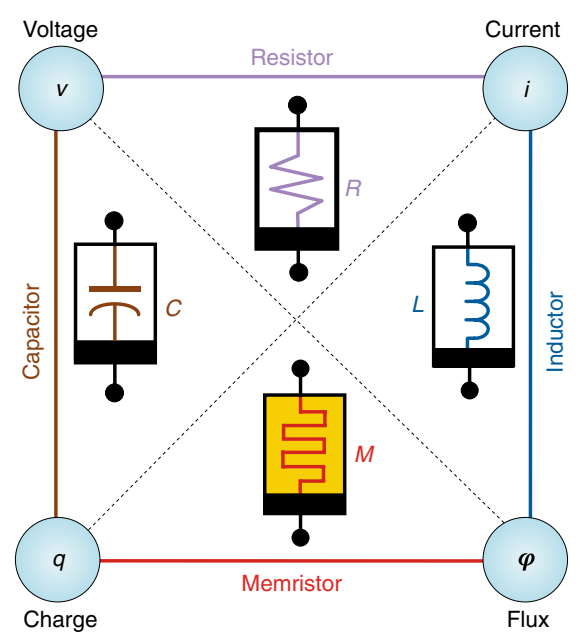

Fig. 1 | The four fundamental two-terminal circuit elements. The resistor is defined by a relationship between voltage $(v)$ and current $(i)$; the inductor by a relationship between flux $(\varphi)$ and current $(i)$; the capacitor by a relationship between charge $(q)$ and voltage $(v)$; and the memristor by a relationship between flux $(\varphi)$ and charge $(q)$.

with the individual electronic devices in the circuit. Thus, basic nonlinear circuit elements must be defined from a black box perspective, independent of their internal composition, material, geometry and architecture.

The basic principles that would predict the memristor were first reported in 1969 in my textbook, Introduction to Nonlinear Network Theory. It then took me a year to derive and prove mathematically the unique circuittheoretic properties and memory attributes of the memristor - the fourth basic circuit element (Fig. 1). On 25 November 1970, I submitted my work, which was entitled "Memristor - the missing circuit element", to the journal IEEE Transactions on Circuit Theory. As I wrote in the paper:

"From the circuit-theoretic point of view, the three basic two-terminal circuit elements are defined in terms of a relationship between two of the four fundamental circuit variables, namely, the current $i$, the voltage $v$, the charge $q$, and the flux-linkage $\varphi$. [...] Three other relationships are given, respectively, by the axiomatic definition of the three classical circuit elements, namely, the resistor (defined by a relationship between $v$ and $i$ ), the inductor (defined by a relationship between $\varphi$ and $i$ ), and the capacitor (defined by a relationship between $q$ and $v$ ). Only one relationship remains undefined, the relationship between $\varphi$ and $q$. From the logical as well as axiomatic points of view, it is necessary for the sake of completeness to postulate the existence of a fourth basic two-terminal circuit element which is characterized by a $\varphi-q$ relationship. This element will henceforth be called the memristor because $[\ldots]$ it behaves somewhat like a nonlinear resistor with memory."

The publication of the paper the following year coincided with my move to the University of California, Berkeley, to spearhead research in a new frontier - nonlinear circuits and systems. Unfortunately, research on the memristor was given a lower priority due to a lack of funding. This was despite recognition by the Institute of Electrical and Electronics Engineers of the potential of the memristor back in 1973, when they awarded me the prestigious W. R. G Baker Award.

For my original 1971 memristor paper, I also built, as a proof of principle, three memristor models using operational amplifiers and off-the-shelf electronic components, which were shown to exhibit three distinct $\varphi-q$ curves on a bespoke memristor curve tracer. But the challenge of fabricating a passive monolithic memristor remained unfulfilled for 37 years. Then, on 1 May 2008, a team of scientists from HP labs, under the leadership of R. Stanley Williams, reported in Nature the first operational monolithic memristor made by sandwiching a thin film of titanium dioxide between platinum electrodes. In addition to providing the physical operating mechanism, the paper by Williams and colleagues also showed a 'pinched hysteresis loop', which is the 'fingerprint' of all memristors.

\section{Leon O. Chua \\ University of California, Berkeley, CA, USA. \\ e-mail:chua@berkeley.edu}

Published online: 14 May 2018

https://doi.org/10.1038/s41928-018-0074-4 